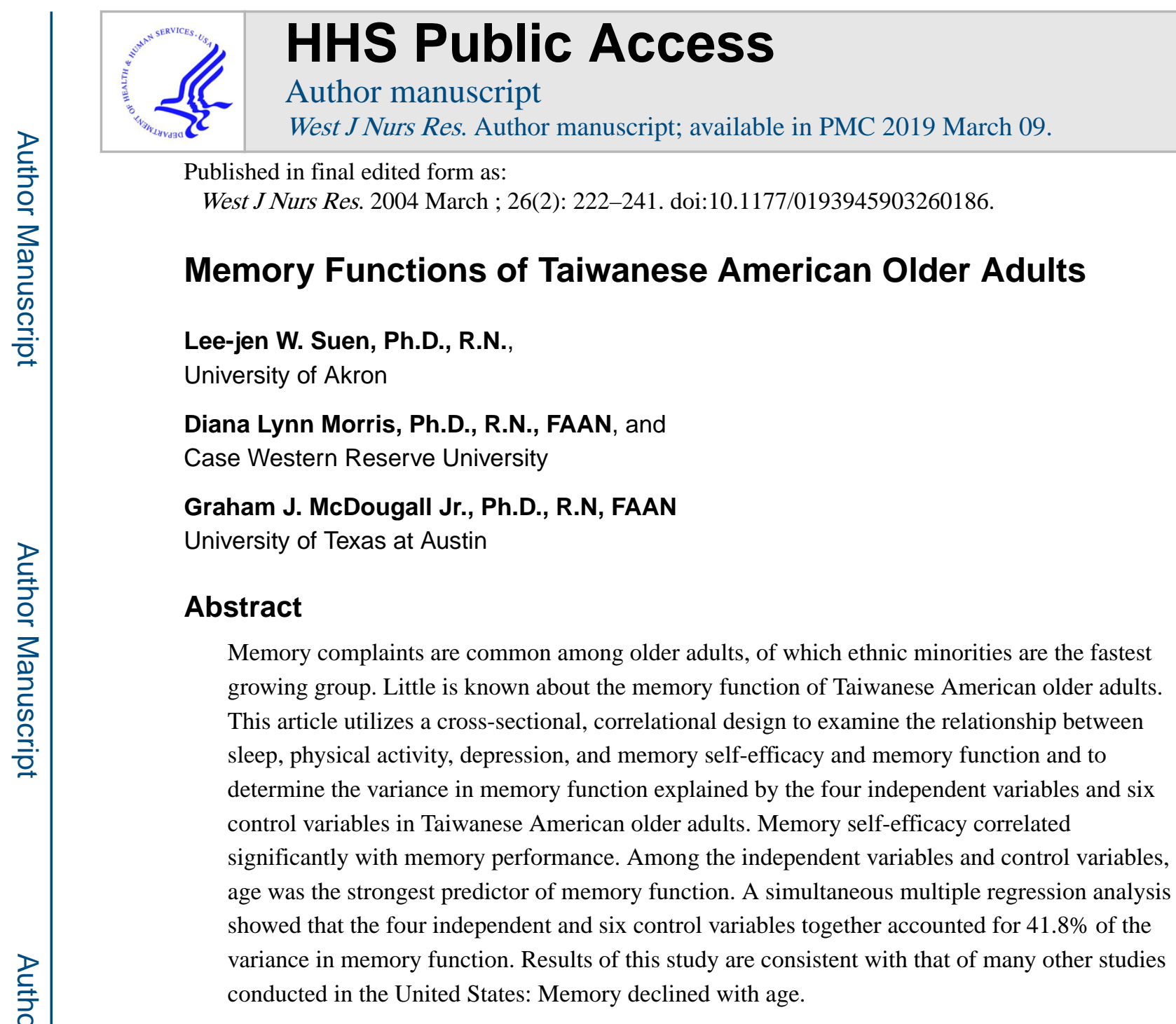

\title{
Keywords
}

memory function; aging; Taiwanese American; Rivermead Memory Test

In the United States, researchers estimated that by 2025, 62.6 million people or $18.5 \%$ of the population will be older than age 65 (U.S. Census Bureau, 2000). Memory complaints are common among older adults (Cutler \& Grams, 1988), and studies have shown that memory performance does, indeed, decline after the age of 50 (Craik \& Jennings, 1992; Light, 1996; Salthouse, 1991). Although literature about aging and memory function is abundant, most memory studies in the United States have been conducted on a White, English-speaking population. Among older adults in the United States, ethnic minority elders are the fastest growing group (Cavanaugh, 1997). Memory decline with aging may not be a universal phenomenon, and factors that affect memory performance may vary across ethnic groups. Taiwanese Americans have not been studied, and their health issues have not been adequately addressed.

\section{MEMORY AND AGING}

Despite the fact that older adults have higher rates of chronic illnesses and declining cognitive functions, they prefer to live independently. Memory is the most important mental 
ability humans possess, and without it, it is difficult to live independently. Without memory, other aspects of cognitive function, such as perception, language comprehension, reasoning, problem solving, planning, and execution, are compromised (Mark, 1992). In spite of certain disabilities, people are often able to maintain their independence when memory function is intact. On the other hand, independence cannot be sustained in the face of memory impairment, regardless of physical capacity.

A national study found that $15 \%$ of people aged 55 and older had reported memory problems within the previous year (Cutler \& Grams, 1988). Memory decline with aging has also been demonstrated by empirical studies (Salthouse, 1991). Memory performance generally declines with age, and older adults, as a group, do not perform memory tasks as well as younger adults; however, interindividual variations are greater within the elderly group than among younger respondents (Lovelace, 1990).

The present study was based on the environmental model of memory performance. It hypothesizes that the degree of memory decrement in elderly persons may be influenced by environmental factors, such as pollutants, disease, nutrition, health practices, and physical fitness (Clarkson-Smith \& Hartley, 1990; Schaie, 1996). Increasing sleep problems (Blazer, Hays, \& Foley, 1995), decreasing physical activities (Kozma \& Stones, 1990), depression (Katz, 1998), and low memory self-efficacy (Ryan \& See, 1993) are often seen in the elderly. All are potentially modifiable factors that may affect memory functions. Knowledge of these factors is necessary to develop effective nursing interventions that can help Taiwanese American elders improve and maintain their memory functions. The present study was designed to examine the relationship between sleep, physical activity, depression, and memory self-efficacy and function in Taiwanese American older adults.

\section{Culture and Memory}

Some cognitive psychologists theorize that each culture has its own characteristic cognitive style (Bateson, 1958/1982). These psychologists have studied the special mnemonic strategies of a given culture hoping to understand the thought and information processing that are typical for a particular culture. In primitive societies, for example, psychologists have observed how preliterate individuals memorized lengthy oral poetry and history (Bateson, 1958/1982; Lord, 1960/1982). In New Guinea, speakers used bundles of leaves as visible, tangible symbols of objects and names during totemic debating, which required great activity of memory. Visual and kinesthetic imagery also appeared to be important mnemonic strategies for the people of this tribe (Bateson, 1958/1982). Dube (1977/1982) found that African groups did much better than U.S. respondents with regard to remembering stories. He attributed this phenomenon to the fact that members of a culture tend to excel in the skills that are encouraged by their culture; that is, many African cultures encourage storytelling. Older persons are expected to tell stories to younger persons, such as parents to their children, or older siblings to younger siblings.

Another way to explore the effect of cultural factors on memory is to examine the environment. For example, Mishra and Singh (1992) hypothesized that to manage life without any sources of light at night would require individuals to keep things in fixed places and remember where they are kept. In their research with the Asur tribe in India, these 
investigators found that intentional and incidental recall for the location is more accurate than recall for "paired" pictures (Mishra \& Singh, 1992). Because of cultural differences, the findings from samples of White older adults may not be applicable to ethnic minorities. Examination of memory and related factors in minority groups has the potential to increase our understanding of memory function in older adults.

\section{Sleep and Memory}

Complaints of decreased sleep satisfaction among older adults (Morgan, 1987) are consistent with polysomnography studies, which found that elders had increased difficulty falling asleep, more frequent nocturnal awakenings, decreased deep sleep, and decreased total actual sleep time (Roffwarg, Muzio, \& Dement, 1966). Increased periodic leg movement at night and sleep apnea (Roth, 1993) contribute to the daytime drowsiness and fatigue of older adults.

When deprived of sleep, people cannot concentrate or remember things; consequently, the effect of sleep on cognitive processing has long been postulated. Humphrey and Kramer (1994) investigated the influence of sleep deprivation on performing automatic and nonautomatic processing tasks. Long-term memory and automatic processing were not affected by sleep deprivation, however, the participants had difficulty learning new information. In another study, insomniacs demonstrated slightly poorer short-term recall on the word-memory test and poorer performance on the one-letter memory test than normal sleepers (Bonnet \& Arand, 1995). Other studies have demonstrated associations between sleep apnea and memory deficit (Bédard, Montplaisir, Richter, Rouleau, \& Malo, 1991; Cohen-Zion et al., 2001; Naëgelé et al., 1995), although a few failed to show any relationship (D. T. R. Berry et al., 1990; Kim et al., 1997; Knight et al., 1987). Differences in the diagnostic criteria for sleep apnea and psychometric methods employed may explain the mixed results. Although the empirical findings are not completely consistent, the majority of the studies demonstrates a significant relationship between sleep and memory.

\section{Physical Activity and Memory}

Researchers have hypothesized that physical exercise improves the maximal oxygen consumption of the body and increases cerebral blood flow, oxidation capacity, and neurotransmitter metabolism. Through these means, exercise prevents degenerative changes in the central nervous system and cognitive capacity (Birren, Woods, \& Williams, 1980; Dustman et al., 1984; Spirduso, 1980). Chodzko-Zajko, Schuler, Solomon, Heinl, and Ellis (1992) examined the influence of physical fitness on memory changes. They found that the high-fitness group performed significantly better than the low-fitness group on the effortful free-recall tests, however, there were no differences on the automatic memory-processing tests.

More rigorous studies have been conducted with experimental design. Stones and Dawe (1993) reported that $30 \mathrm{~min}$ of low-intensity aerobic activity had positive effects on the neuropsychological performance of nursing home residents. An intensive aerobic training for 9 to 12 months, in a sample of older adults, did not improve short-term memory or their scores on the Wechslers Memory Scale (WMS) (Hill, Storandt, \& Malley, 1993). The 
control group, however, showed a significant decline in WMS logical memory, suggesting that although exercise does not lead to improved memory performance, it may help prevent memory decline.

\section{Depression and Memory}

The research results concerning the effects of depression on memory are mixed. Some researchers have found depression associated with impairment of visuospatial recognition memory (Rubinsztein, Michael, Paykel, \& Sahakian, 2000), decreased free recall and normal-cued recall and recognition (Fossati, Coyette, Ergis, \& Allilaire, 2002), and impairment in short-term memory (Glass, Uhlenhuth, \& Weinreb, 1978). Other research showed no negative effects of depression on automatic memory performance (MacQueen, Galway, Hay, Young, \& Joffe, 2002). The discrepancy in findings may be due to differences in research designs, selection of participants, depression measures, and memory tasks.

To synthesize knowledge from the research literature, two meta-analyses have been conducted. Burt, Zembar, and Niederehe (1995) combined data from 147 studies published from 1967 to 1991 on adults of all ages. These investigators found that depression is associated in small to moderate degrees with specific aspects of memory. In addition, memory impairment is more likely to occur in inpatients than in outpatients. Kindermann and Brown (1997) conducted a meta-analysis of depression and memory research based on 40 studies published between 1973 and 1994, with samples restricted to participants age 55 years and older. Most of the studies showed moderate effect size, with larger effect sizes found in older groups. Depression seems to have some effect on memory. The effect does vary based on the participant's age, the severity of depression, and the specific memory tasks participants are asked to perform.

\section{Memory Self-Efficacy and Memory}

Memory self-efficacy has been found to be related to memory loss in elderly people (Cavanaugh \& Green, 1990; McDougall, 1999; West \& Berry, 1994). Memory self-efficacy refers to the belief in one's ability to organize and master a particular memory task (Cavanaugh, Feldman, \& Hertzog, 1998). According to Bandura (1997), a person with strong memory self-efficacy is motivated to engage in deeper levels of information processing and puts more cognitive effort into memory tasks, such as mental rehearsal and elaborate coding, than a person with low memory self-efficacy. Studies have shown that older adults tend to have lower memory self-efficacy than younger adults (Hertzog, Dixon, Schulenberg, \& Hultsch, 1987; Luszcz, 1993; McDougall, 1995; West \& Berry, 1994).

Empirical studies have had mixed results with regard to memory self-efficacy and memory performance. Four studies have shown that memory self-efficacy was significantly associated with everyday and laboratory memory performance (J. M. Berry, West, \& Dennehey, 1989; Luszcz, 1993; McDougall, 1999; Rebok \& Balcerak, 1989). Levy (1996) demonstrated that those influenced by a positive image of aging experienced improved memory self-efficacy and memory performance, whereas a negative stereotype tended to decrease memory self-efficacy and memory performance. Seeman, Rodin, and Albert (1993) found that memory self-efficacy was not related to memory performance; anxiety and 
motivation during testing were more important factors. Ponds and Jolles (1996) found that memory complaints were related to low memory self-efficacy but not to poor memory performance. The mixed results may be due to differences in sample selection, operationalization of memory self-efficacy, and memory tasks.

\section{PURPOSE}

The purpose of this cross-sectional, correlational study was to examine the memory performance of Taiwanese American older adults and to investigate the relationships among sleep, physical activity, depression, and memory self-efficacy and performance. In this study, memory function is the dependent variable. It is measured by memory performance and used interchangeably with that term. Sleep, physical activity, depression, and memory selfefficacy are independent variables.

\section{METHOD}

\section{Design}

In this study, a cross-sectional design was used. Memory function was measured by a memory test. Sleep, physical activity, depression, and memory self-efficacy were assessed through standardized instruments.

\section{Sample and Setting}

The target population for the study was Taiwanese Americans aged 60 years or older, who were living in the community. The convenience sample was recruited from a Taiwan Center in a city in the northeastern United States. Snowballing was also used to recruit additional participants. The Taiwan Center is a community center open to all people, however, the majority of its members are Taiwanese immigrants. Sample size was calculated through power analysis (Cohen, 1988). A medium effect size was used, $F^{2}=.15$, with alpha (Type I error) set at .05 , and power was set at .8. The desired sample size for the research was 95 . The resultant sample consisted of 100 participants, 47 women, and 53 men. Excluded from the study were those with a history of memory disorders or dementia, as reported by the participant or the participant's family, those with hearing or vision problems, and those who made four or more mistakes on the Short Portable Mental Status Questionnaire (SPMSQ) (Pfeiffer, 1975). Only one participant made more than four mistakes on the SPMSQ and was excluded from the study. Due to the assumptions of the statistical analysis, participants were required to be independent from each other; thus, none of the participants' spouses or siblings were allowed to participate.

Participants' $(N=100)$ ages ranged from 60 to 88 years $(M=67.4, S D=7.0)$, years of education ranged from 0 to $27(M=14.5, S D=5.7)$; and the average U.S. residency was 22.8 years. Of the participants, $79 \%$ were married, $36 \%$ were agnostic, $29 \%$ Christian, and $24 \%$ Buddhist. Of the participants, $30 \%$ were still working full-time and $9 \%$ part-time. Sixty-six percent were proficient in English. From the entire sample, $88 \%$ stated that their memory had declined, and $56 \%$ used some sort of memory aide, such as a notepad or a personal organizer, to help them remember important information. 


\section{Data Collection Procedure}

The University's Institutional Review Board and the Taiwan Center approved this study. Because memory performance can only be tested by face-to-face, structured interviews, all other information was collected through face-to-face interviews. Data were collected in Taiwanese by the investigator only. Interviews were done either at the Taiwan Center, or at participants' homes. After obtaining consent from participants, the investigator collected demographic and mental status data to check for eligibility. If a participant was eligible for the study, the interview continued. To limit the possibility that the participants might change on their memory self-efficacy ratings after taking the memory test, the Memory SelfEfficacy Questionnaire (MSEQ) (J. M. Berry et al., 1989) was administered before the Extended Rivermead Behavioural Memory Test (ERBMT) (de Wall, Wilson, \& Baddeley, 1994). The average length of an interview was $65 \mathrm{~min}$.

\section{Instruments}

The English version of the instruments was translated into Taiwanese by a team of bilingual Taiwanese Americans with either Ph.D. or M.D. degrees, paying special attention to the semantic and technical equivalence of each item. The final version of Taiwanese instruments was pretested on five Taiwanese Americans for clarity and comprehensibility. The questionnaires and memory function tests used in the present research measure the four independent variables (sleep, depression, memory self-efficacy, and physical activity) and the dependent variable, memory performance. Personal background data (age, education, and gender) and control variables (medical illness and medication use) were included.

The SPMSQ was used to screen cognitive function. This instrument was developed by Pfeiffer (1975) to detect the presence of cognitive impairment. The SPMSQ consists of 10 questions that test for orientation, memory, and serial mental operations. Each question is worth one point, with three to four errors indicating mild intellectual impairment. Black participants are allowed one more error, however, there are no specifications for other minority ethnic groups. The reliability of the SPMSQ was established by test-retest reliability at .82 . Evidence of criterion validity showed a $92 \%$ agreement between SPMSQ scores and the clinical diagnose (Pfeiffer, 1975).

Regarding illness and medication use, participants were asked to list any illness episodes that required visits to a physician during the last year and any chronic afflictions, such as hypertension or diabetes. Participants were asked to list any prescription and nonprescription drugs that they were currently taking. In the data analysis, illness referred to the total number of illnesses reported. Medication use was likewise determined by the number of drugs reported; medication use was divided into drugs used for health promotion (vitamins and supplements) and drugs used for treatment (prescription and over-the-counter drugs). For data analysis, gender was coded as male $=1$ and female $=0$.

The Pittsburgh Sleep Quality Index (PSQI) was used to measure sleep, subjective sleep quality, and sleep disturbances (Buysse, Reynolds, Monk, Berman, \& Kupfer, 1989). The PSQI consists of 19 questions, which evaluate sleep quality, sleep latency, sleep duration, habitual sleep efficiency, sleep disturbances, use of sleeping medication, and daytime 
dysfunction. All seven component scores are weighted equally on a 0 to 3 scale, with higher scores indicating worse sleep quality. The reliability of the PSQI was reported by test-retest reliability $(r=.85)$, and internal consistency estimates (alpha $=.83)$. Construct validity was established by comparing extreme groups (Buysse et al., 1989). In the present study, the internal consistency reliability was .82 .

The Physical Activity Questionnaire (PAQ) was used to measure physical activity. Baecke, Burema, and Frijters (1982) originally developed the PAQ for young adults. Voorrips,

Ravelli, Dongelmans, Deurenberg, and van Staveren (1991) modified the questionnaire for an elderly population. The questionnaire does not include occupational activity. Respondents are asked about household activities, sports, and other leisure-time activities over the last year. The three subset scores are summed to obtain one activity score, with higher scores indicating greater physical activity. The test-retest reliability of the PAQ was .89 . The criterion-related validity was .72 and .78 (Voorrips et al., 1991). Cronbach's alpha was .62 for the current research.

The Geriatric Depression Scale (GDS) was used to measure the presence of depression symptoms in this study (Yesavage et al., 1983). The GDS is a self-report questionnaire with all questions in a yes or no format. The theoretical range of the GDS is from 0 to 30 , with higher scores indicating the presence of more depressive symptoms. Test-retest reliability was .85 , split-half reliability was .94 , Cronbach's alpha was .94 , and concurrent validity was .84 (Yesavage et al., 1983). Cronbach's alpha was .83 for this sample.

The current study used the Memory Self-Efficacy Questionnaire (MSEQ) to measure memory self-efficacy, which refers to one's belief that one will be able to perform a specific memory task. The MSEQ was developed byJ. M. Berry et al. (1989) and is based on Bandura's (1986) self-efficacy theory. Participants were asked to indicate their ability to perform 10 memory tasks: recalling grocery lists, locations of items, telephone numbers, words, digits, directions on a map, errands, mazes, and recognizing pictures and photographs. The test-retest correlation was .89 . The internal consistency was calculated by Cronbach's alpha coefficient at .85 . Predictive validity was established using the canonical correlation with $R_{\mathrm{c}}=.59$ and Wilks's $\lambda=.52$ (J. M. Berry et al., 1989). Cronbach's alpha was .90 for this sample.

The Rivermead Behavioural Memory Test (RBMT) (Wilson, Cock-burn, \& Baddeley, 1985) is an objective measurement of everyday memory, that is, the memory skills necessary for functioning in normal life. The RBMT consists of four parallel tests (A, B, C, and D), each with 12 test components. The 12 subtests include first name, last name, story (immediate and delayed), hidden belonging, appointment, route (immediate and delayed), message, faces, object pictures, and orientation. The reliability of the RBMT was established by parallel-form reliability (Wilson, Cockburn, Baddeley, \& Hiorns, 1991). The correlations between Version A and Versions B, C, and D were .86, .83, and .88, respectively. Construct validity was evaluated as the correlation (.75) between the RBMT scores and the number of memory lapses (Wilson et al., 1991). The RBMT was originally designed to detect memory impairment in patients with brain damage, therefore a ceiling effect may occur for normal adults. To adjust for this effect, de Wall et al. (1994) made the test more difficult by doubling 
the testing material. Versions $\mathrm{A}$ and $\mathrm{B}$ were combined, as were versions $\mathrm{C}$ and $\mathrm{D}$, to form the Extended Rivermead Behavioural Memory Test (ERBMT), which was used in the current research. Some adjustments were made on the ERBMT to measure memory function in Taiwanese persons. For name recall, the investigator used one Taiwanese name and one English name, instead of two English names. The same was true for faces: five Asian faces and five Caucasian faces were used, instead of 10 Caucasian faces. Cronbach's alpha was . 78 for the present study.

\section{Data Analysis}

Descriptive statistics were used to describe the magnitude of each variable, and inferential statistics (correlation coefficient and multiple regression) were performed to examine the relationship between dependent and independent variables. The investigator explored the variables for outliers and influential data and examined assumptions of multiple regression analysis for potential violations. The internal consistency reliability of the scales was estimated by the Cronbach's alpha coefficient for each instrument.

\section{RESULTS}

Table 1 presents the 12 subscale scores and total scores for the memory test. Among all subscales of the ERBMT, many obtained a perfect score on 20-object recognition (38\%), recall of a 5-point route (88\%), two messages (87\%), and orientation (76\%). Face, name, story, and appointment tasks, however, seemed to be more difficult for the elderly Taiwanese Americans to remember, and variations within the sample were wider.

Regarding the four independent variables, average value of the PSQI was6.0, where 5 or higher indicates poor sleep quality. Thus, overall Taiwanese American older adults in this sample had poor sleep quality. There was a very wide range of physical activity; some participants hardly exercised, while others played tennis and climbed mountains $(M=9.0$, $S D=4.9)$. The scores of the GDS ranged from 0 to $19(M=4.97, S D=4.26)$. If 11 is used as the cutoff score for screening depression, then $9 \%$ of the sample experienced some depression. The average of memory self-efficacy level in 10 tasks ranged from .5 to 5 ( $M=$ 2.46, $S D=.88$ ). Among the 10 tasks measured by the MSEQ, the Taiwanese American elders had the highest confidence level in location memory and the lowest in digit memory. Confidence for photograph memory was low as well.

Hypertension (37\%) was the most common health problem, followed by arthritis (19\%), pain $(18 \%)$, diabetes (10\%), high cholesterol (10\%), and heart disease (6\%). The most frequently taken type of drug was antihypertension agents (28\%), followed by nonsteroidal anti-inflammatory drugs (NSAIDs) (9\%), lipid-lowering medications (8\%), pain medications (7\%), and diabetic medications (5\%). It is noted that although some participants reported having health problems, they did not always use medication for treatment. Elderly Taiwanese Americans used more than 13 different kinds of health-promotion drugs. Of the participants, $63 \%$ were taking multivitamins, $45 \%$ vitamin E, and $42 \%$ calcium. Fourteen percent were taking Gingko Biloba in hopes of improving or maintaining their memory function, but they did not notice any effects from the herb. 
Table 2 presents the correlation coefficients of the predictor variables and the dependent variable. To test the individual relationships of the independent variables (sleep, physical activity, depression, and memory self-efficacy), and demographic and control variables (age, gender, years of education, total number of illnesses, total number of treatment drugs, and total number of health-promotion drugs) with the dependent variable, Pearson's correlation coefficients were calculated. Among the four independent variables, only memory selfefficacy was significantly correlated with memory function $(r=.33, p<.01)$. The higher the memory self-efficacy score, the better the memory function. Among all control variables, age $(r=-.54, p<.001)$ and total number of illnesses $(r=-.34, p<.001)$ had negative correlations with memory performance; whereas education $(r=.46, p<.001)$ and number of health-promotion medications used $(r=.26, p<.05)$ had positive relationships with memory performance. Memory function correlated most strongly with age $(r=-.54, p<.001)$, and as age increased, memory performance decreased.

Table 3 shows the results of simultaneous multiple regression for the four independent variables regressed on memory function, and the simultaneous regression analysis with the four independent variables, and six control variables regressed on memory performance. The four independent variables accounted for $13 \%(F=3.50, p<.01)$ of the total variance of memory function in the elderly Taiwanese Americans. Memory self-efficacy was the only predictor with a significant effect on memory function $(\beta=.31, p=.002)$. All 10 predictors together accounted for $42 \%(F=6.39, p<.001)$ of the variance in ERBMT performance. Among the 10 predictors, only age $(\beta=-.38, p<.001)$ and the number of health-promotion drugs $(\beta=.21, p<.01)$ had a significant effect on memory performance. None of the four independent variables showed any significant effect on memory function when all other predictors were held constant. Among the control variables, education, gender, and number of chronic illnesses, correlated significantly with memory function (Table 3) but failed to show any significant effect on memory function in this multiple regression model.

\section{DISCUSSION}

This study examined the relationship between sleep, physical activity, depression, and memory self-efficacy and function and determined the predictive ability of the independent variables on memory function. The results showed that memory self-efficacy was positively correlated with memory performance $(r=.33, p<.01)$. This finding is consistent with Bandura's (1997) theory of memory self-efficacy, which states that a person with high memory self-efficacy will try harder to meet memory demands. Other investigations have found significant positive relationships between memory self-efficacy and memory performance (Luszcz, 1993; McDougall, 1999; Rebok \& Balcerak, 1989). It is possible that people with better memory function have more confidence in their memory. Bandura's theory further purports that cultural stereotyping, social judgments, and personal experiences all contribute to a person's self-efficacy. In Taiwanese culture, age may imply senility, stubbornness, and regression to a childlike mentality, as well as wisdom and rich life experiences. There are no systematic investigations examining the effect of cultural beliefs on memory efficacy in Taiwanese American older adults. 
Three independent variables sleep, depression, and physical activity failed to show significant correlations with memory function. Because the PSQI asks for general sleep quality within the last month, the effects of poor sleep quality on memory function may not be as obvious as in the acute sleep deprivation of experimental studies. It is difficult to compare the present study results with previous studies, because subjective sleep quality and memory have not been measured in the same manner. Because only $9 \%$ of participants experienced mild to moderate depressive symptoms in the present sample, it was difficult to detect the effect of depression on memory function. Many ethnic minorities living in the United States anticipate increased isolation during their senior years, and the later years of life can be stressful for them. Study participants reside in a city where Taiwanese American older adults can function without speaking English, where public transportation is very convenient, and the Taiwan Center provides a place for them to socialize. Unlike those in other towns and cities, the Taiwanese Americans in this study may not experience the cultural stress that leads to increased depression in old age. The PAQ measures household, leisure, and sport activities and does not include items for work or occupational activity. Of the participants in the current study, 30\% were still working full-time, and $9 \%$ were working part-time. Work-related physical activities, therefore, were not measured.

The demographic and control variables of age, gender, education, total number of illnesses, and total number of health-promotion drugs correlated significantly with memory function. Among the six control variables, age has the highest inverse correlation with memory performance $(r=-.54)$. This relationship between age and memory function is consistent with the results of many studies (Christensen et al., 1994; Cockburn \& Smith, 1991; Mejia, Pineda, Alvarez, \& Ardila, 1998; Salthouse, Kausler, \& Saults, 1990). In spite of the participants' being Taiwanese American, the results are similar to other studies in older Americans.

The results of the multiple regression showed that sleep, depression, physical activity, and memory self-efficacy accounted for a significant portion of the variance (13\%) in the memory function of Taiwanese American older adults. Memory self-efficacy, however, was the only predictor that had a significant effect on memory performance. The four independent variables and six demographic and control variables accounted for $42 \%$ of the variance in memory function. With 10 predictors, only age and the total number of healthpromotion drugs had a significant effect on memory function. Memory self-efficacy, education, gender, and number of illnesses did not have a significant effect on memory function when other variables were held constant in the multiple regression analysis. These variables were not significant because of their correlations with age. As a result, the effect of memory self-efficacy, education, gender, and number of illnesses on memory performance was greatly minimized.

When compared to their male counterparts in this sample, women were older, had lower education attainment, lower memory self-efficacy, higher number of illnesses, and lower memory performance. Age remained the strongest factor affecting memory function in the multiple regression analysis. Salthouse et al. (1990) and Christensen et al. (1994) found a significant decline in memory across age groups, even when gender, education, and health were controlled. Cockburn and Smith (1991), who used the RBMT to measure memory, also 
found that age was the most significant predictor of the overall Rivermead score and that it was more important than gender, marital status, eyesight, hearing, recent illnesses, self-rated anxiety, depression, drugs currently taken, and social support.

The total number of health-promotion drugs was the only other predictor to retain a significant effect on memory performance in the multiple regression analysis. From this cross-sectional study, it cannot be determined whether the total number of health-promotion drugs used affects memory. It is possible that participants who took more health-promotion drugs had healthier lifestyles or those who could remember taking their health-promotion drugs, and actually reported it, had a better memory in the first place.

Several study limitations need to be kept in mind when interpreting results and discussing implications. Using a convenience sample limits the generalizability of the results. The present sample was generally healthy, had no serious physical and mental health problems, and were active in a Taiwanese community in the United States. Another limitation of this study is that the physical activity measurements may not have been sensitive to the full range of potential activities in which elders were engaged. As participants were required to recall events over the entire last year, there is the risk that recalled data was susceptible to report errors. Certain illnesses are more likely to affect memory than others. For the purpose of this study, the total number of illnesses was used in analysis. A larger sample would be needed to examine the relationship between a specific illness and memory function.

The present study contributed essential data on this little-studied ethnic minority population. This study increases our understanding of memory function related to sleep, depression, physical activity, memory self-efficacy, aging, illness, and medication uses of Taiwanese American older adults. Memory decline with age in Taiwanese Americans was consistent with findings from most studies of memory in older adults in the United States.

\section{Acknowledgments}

We acknowledge the National Institute of Nursing Research for providing the Minority Supplement Grant (NR-04204-01S1) to support this research.

\section{REFERENCES}

Baecke JAH, Burema J, \& Frijters JER (1982). A short questionnaire for the measurement of habitual physical activity in epidemiological studies. American Journal of Clinical Nutrition, 36, 936-941. [PubMed: 7137077]

Bandura A (1986). Social foundations of thought and action: A social cognitive theory. Englewood Cliffs, NJ: Prentice Hall.

Bandura A (1997). Self-efficacy: The exercise of control. New York: Freeman.

Bateson G (1982). Totemic knowledge in New Guinea In Neisser U (Ed.), Memory observed: Remembering in natural contexts (pp. 269-273). San Francisco: Freeman (Original work published 1958)

Bédard M, Montplaisir J, Richter F, Rouleau I, \& Malo J (1991). Obstructive sleep apnea syndrome: Pathogenesis of neuropsychological deficits. Journal of Clinical Experimental Neuropsychology, 13, 950-964. [PubMed: 1779033]

Berry DTR, Phillips BA, Cook YR, Schmitt FA, Honeycutt NA, Arita AA, et al. (1990). Geriatric sleep apnea syndrome: A preliminary description. Journal of Gerontology: Medical Sciences, 45(5), M169-M174. 
Berry JM, West RL, \& Dennehey DM (1989). Reliability and validity of the memory self-efficacy questionnaire. Developmental Psychology, 25(5), 701-713.

Birren JE, Woods AM, \& Williams MV (1980). Behavioral slowing with age: Causes, organization, and consequences In Poon LW (Ed.), Aging in the 1980s: Psychological issues (pp. 293-308). Washington, DC: American Psychological Association.

Blazer DG, Hays JC, \& Foley DJ (1995). Sleep complaints in older adults: A racial comparison. Journal of Gerontology: Medical Science, 50A(5), M280-M284.

Bonnet MH, \& Arand DL (1995).24-hourmetabolic rate in insomniacs and matched normal sleepers. Sleep, 18(7), 581-588. [PubMed: 8552929]

Burt DB, Zembar MJ, \& Niederehe G (1995). Depression and memory impairment: A meta-analysis of the association, its pattern, and specificity. Psychological Bulletin, 117(2), 285-305. [PubMed: 7724692]

Buysse DJ, Reynolds CF, Monk TH, Berman SR, \& Kupfer DJ (1989).The Pittsburgh Sleep Quality Index: A new instrument for psychiatric practice and research. Psychiatry Research, 28, 193-213. [PubMed: 2748771]

Cavanaugh JC (1997). Adult development and aging (3rd ed.). Pacific Grove, CA: Brooks/Cole.

Cavanaugh JC, Feldman JM, \& Hertzog C (1998). Memory beliefs as social cognition: A reconceptualization of what memory questionnaires assess. Review of General Psychology, 2(1), $48-65$.

Cavanaugh JC, \& Green EE (1990). I believe, therefore I can: Self-efficacy beliefs in memory aging In Lovelace EA (Ed.), Aging and cognition: Mental processes, self-awareness and interventions (pp. 189-230). Amsterdam: North-Holland.

Chodzko-Zajko WJ, Schuler P, Solomon J, Heinl B, \& Ellis NR (1992). The influence of physical fitness on automatic and effortful memory changes in aging. International Journal of Aging and Human Development, 35(4), 265-285. [PubMed: 1428192]

Christensen H, Mackinnon A, Jorm AF, Henderson AS, Scott LR, \& Korten AE (1994). Age differences and interindividual variation in cognition in community-dwelling elderly. Psychology and Aging, 9(3), 381-390. [PubMed: 7999323]

Clarkson-Smith L, \& Hartley AA (1990). Structural equation models of relationships between exercise and cognitive abilities. Psychology and Aging, 5(3), 437-446. [PubMed: 2242248]

Cockburn J, \& Smith PT (1991). The relative influence of intelligence and age on everyday memory. Journal of Gerontology: Psychological Sciences, 46(1), P31-P36.

Cohen J (1988). Statistical power analysis for the behavioral sciences (2nd ed.). Hillsdale, NJ: Lawrence Erlbaum.

Cohen-Zion M, Stepnowsky C, Marler, Shochat T, Kripke DF, \& Ancoli-Israel S (2001). Changes in cognitive function associated with sleep disordered breathing in older people. Journal of American Geriatric Society, 49(12), 1622-1627.

Craik FIM, \& Jennings JM (1992). Human memory In Craik FIM \& Salthouse TA (Eds.), The handbook of aging and cognition (pp. 51-110).Hillsdale, NJ: Lawrence Erlbaum.

Cutler SJ, \& Grams AE (1988). Correlates of self-reported everyday memory problems. Journal of Gerontology: Social Sciences, 43(3), S82-S90.

de Wall C, Wilson BA, \& Baddeley AD (1994). The extended Rivermead Behavioural Memory Test: A measure of everyday memory performance in normal adults. Memory, 2(2), 149-166. [PubMed: 7584289]

Dube EF (1982). Literacy, cultural familiarity, and intelligence as determinants of story recall In Neisser U (Ed.), Memory observed: Remembering in natural contexts (pp. 274-292). San Francisco: Freeman (Original work published 1977)

Dustman RE, Ruhling RO, Russell EM, Shearer DE, Bonekat HW, Shigeoka JW, et al. (1984). Aerobic exercise training and improved neuropsychological function of older individuals. Neurobiology of Aging, 5, 35-42. [PubMed: 6738784]

Fossati P, Coyette F, Ergis AM, \& Allilaire JF (2002).Influence of age and executive functioning on verbal memory of inpatients with depression. Journal of Affect Disorder, 68(2/3), 261-271.

Glass RM, Uhlenhuth EH, \& Weinreb H (1978). Imipramine-reversible deficit in out-patient depressives. Psychopharmacology Bulletin, 14, 10-12. [PubMed: 349595] 
Hertzog C, Dixon RA, Schulenberg JE, \& Hultsch D (1987). On the differentiation of memory beliefs from memory knowledge: The factor structure of the metamemory in adulthood scale. Experimental Aging Research, 13(2), 101-107. [PubMed: 3678344]

Hill RD, Storandt M, \& Malley M (1993). The impact of long-term exercise training of psychological function in older adults. Journal of Gerontology: Psychological Sciences, 48(1), P12-P17.

Humphrey DG, \& Kramer A (1994). Influence of extended wakefulness on automatic and nonautomatic processing. Human Factors, 36(4), 652-669. [PubMed: 7875692]

Katz IR (1998). Depression as a pivotal component in secondary aging: Opportunities for research, treatment and prevention In Lomranz J (Ed.), Handbook of aging and mental health: An integrative approach (pp. 462-482). New York: Plenum.

Kim HC, Young T, Matthews CG, Weber SM, Woodard AR, \& Palta M (1997). Sleep-disordered breathing and neuropsychological deficits. American Journal of Respiratory Critical Care Medicine, 156, 1813-1819. [PubMed: 9412560]

Kindermann SS, \& Brown GG (1997). Depression and memory in the elderly: A meta-analysis. Journal of Clinical and Experimental Neuropsychology, 19(5), 625-642. [PubMed: 9408794]

Knight H, Millman RP, Gur RC, Saykin AJ, Doherty JU, \& Pack AI (1987). Clinical significance of sleep apnea in the elderly. Annual Review of Respiratory Disease, 136, 845-850.

Kozma A, \& Stones MJ (1990). Decrements in habitual and maximal physical performance with age In Perlmutter M (Ed.), Late life potential (pp. 1-23). Washington, DC: Gerontological Society of America.

Levy B (1996). Improving memory in old age through implicit self-stereotyping. Journal of Personality and Social Psychology, 71(6), 1092-1107. [PubMed: 8979380]

Light LL (1996). Memory and aging In Bjork EL \& Bjork RA (Eds.), Memory (2nd ed., pp. 443-490). San Diego: Academic Press.

Lord AB (1982). Oral poetry in Yugoslavia In Neisser U (Ed.), Memory observed: Remembering in natural contexts (pp. 243-257). San Francisco: Freeman (Original work published 1960)

Lovelace EA (1990). Cognitive aging: A summary overview In Lovelace EA (Ed.), Aging and cognition: Mental processes, self-awareness, and interventions (pp. 407-434). Amsterdam: NorthHolland.

Luszcz MA (1993). When knowing is not enough: The role of memorybeliefs in prose recall of older and younger adults. Australian Psychologist, 28(1), 16-20.

MacQueen GM, Galway TM, Hay J, Young LT, \& Joffe RT (2002). Recollection memory deficits in patients with major depressive disorder predicted by past depressions but not current mood state or treatment status. Psychological Medicine, 32(2), 251-258. [PubMed: 11866320]

Mark VH (with Mark JP). (1992). Reversing memory loss: Proven methods for regaining, strengthening, and preserving your memory. Boston: Houghton Mifflin.

McDougall GJ (1995). Memory self-efficacy and strategy use in successful elders. Educational Gerontology, 21, 357-373.

McDougall GJ (1999). Cognitive interventions among older adults. Annual Review of Nursing Research, 17, 219-240.

Mejia S, Pineda D, Alvarez LM, \& Ardila A (1998). Individual differences in memory and executive function ability during normal aging. Internal Journal of Neuroscience, 95, 271-284.

Mishra RC, \& Singh T (1992). Memories of Asur children for locations and pairs of pictures. Psychological Studies, 37, 38-46.

Morgan K (1987). Sleep and aging: A research-based guide to sleep in later life. Baltimore: Johns Hopkins University Press.

Naëgelé B, Thouvard V, Pépin J, Lévy P, Bonnett C, Perret JE, et al. (1995). Deficits of executive cognitive functions in patients with sleep apnea syndrome. Sleep, 18, 43-52. [PubMed: 7761742]

Pfeiffer E (1975). A Short Portable Mental Status Questionnaire for the assessment of organic brain deficit in elderly patients. Journal of the American Geriatrics Society, 23, 433-441. [PubMed: 1159263]

Ponds RWHM, \& Jolles J (1996).Memory complaints in elderly people: The role of memory abilities, metamemory, depression, and personality. Educational Gerontology, 22, 341-357. 
Rebok GW, \& Balcerak LJ (1989). Memory self-efficacy and performance differences in young and old adults: The effect of mnemonic training. Developmental Psychology, 25(5), 714-721.

Roffwarg HP, Muzio JN, \& Dement WC (1966). Ontogenetic development of the human sleep-dream cycle. Science, 152, 604-619. [PubMed: 17779492]

Roth T (1993). Sleep in the elderly: A clinical challenge In Albarede JL, Morley JE, Roth T, \& Vellas BJ (Eds.), Sleep disorders and insomnia in the elderly (pp. 7-9). New York: Springer.

Rubinsztein JS, Michael A, Paykel ES, \& Sahakian BJ (2000). Cognitive impairment in remission in bipolar affective disorder. Psychological Medicine, 30(5), 1025-1036. [PubMed: 12027040]

Ryan EB, \& See SK (1993). Age-based beliefs about memory changes for self and others across adulthood. Journal of Gerontology: Psychological Sciences, 48(4), 199-201.

Salthouse TA (1991). Theoretical perspectives on cognitive aging. Hillsdale, NJ: Lawrence Erlbaum.

Salthouse TA, Kausler DH, \& Saults JS (1990). Age, self-assessed health status, and cognition. Journal of Gerontology: Psychological Sciences, 45(4), P156-P160.

Schaie KW (1996). Lifestyle variables that affect intellectual functioning In Schaie KW, Intellectual development in adulthood: The Seattle Longitudinal Study (pp. 265-277). New York: Cambridge University Press.

Seeman TE, Rodin J, \& Albert M (1993). Self-efficacy and cognitive performance in high-functioning older individuals. Journal of Aging and Health, 5(4), 455-474.

Spirduso WW (1980). Physical fitness, aging, and psychomotor speed: A review. Journal of Gerontology, 35, 850-865. [PubMed: 7002994]

Stones MJ, \& Dawe D (1993). Acute exercise facilitates semantically cued memory in nursing home residents. Journal of the American Geriatric Society, 41, 531-534.

U.S. Census Bureau. (2000). United States Census Bureau. DP-1. Profile of general demographic characteristics: 2000. Available from http://factfinder.census.gov/servlet/QTTable? ds_name=DEC_2000_SF1_U\&geo_id=01000US\&

Voorrips LE, Ravelli ACJ, Dongelmans PCA, Deurenberg P, \& van Staveren WA (1991). A physical activity questionnaire for the elderly. Medicine and Science in Sports and Exercise, 23(8), 974979. [PubMed: 1956274]

West RL, \& Berry JM (1994). Age declines in memory self-efficacy: General or limited to particular tasks and measures? In Sinnott JD (Ed.), Interdisciplinary handbook of adult lifespan learning (pp. 426-455). Westport, CT: Greenwood.

Wilson BA, Cockburn J, \& Baddeley AD (1985). The Rivermead Behavioural Memory Test. Bury St. Edmunds, UK: Thames Valley Test Company.

Wilson BA, Cockburn J, Baddeley AD, \& Hiorns R (1991). The Rivermead Behavioural Memory Test Supplement Two (2nd ed.). Bury St. Edmunds, UK: Thames Valley Test Company.

Yesavage JA, Brink TL, Rose TL, Lum O, Huang V, Adey M, et al. (1983). Development and validations of a geriatric depression screening scale: A preliminary report. Journal of Psychiatric Research, 17(1), 37-49.

West J Nurs Res. Author manuscript; available in PMC 2019 March 09. 
TABLE 1:

Total Score and Subscales Score of the Extended Rivermead Behavioural Memory Test $(N=100)$

\begin{tabular}{|c|c|c|c|}
\hline Variable & M (SD) & Range & Theoretical Range \\
\hline Story immediate & $8.21(3.17)$ & $2-18$ & $0-21$ \\
\hline Story delayed & $7.13(3.02)$ & $0-16$ & $0-21$ \\
\hline Picture & $18.4(2.03)$ & $8-20$ & $0-20$ \\
\hline \multicolumn{4}{|l|}{ Face } \\
\hline Asian face & $3.70(1.28)$ & $0-5$ & $0-5$ \\
\hline Western face & $3.12(1.55)$ & $0-5$ & $0-5$ \\
\hline Route immediate & $4.85(.42)$ & $3-5$ & $0-5$ \\
\hline Route delayed & $4.90(.33)$ & $3-5$ & $0-5$ \\
\hline Message immediate & $4.35(.78)$ & $2-5$ & $0-5$ \\
\hline Message delayed & $4.84(.49)$ & $3-5$ & $0-5$ \\
\hline Orientation & $12.76(.43)$ & $11-13$ & $0-13$ \\
\hline \multicolumn{4}{|l|}{ Name (2 names) } \\
\hline Taiwanese name & $2.97(1.15)$ & $0-4$ & $0-4$ \\
\hline American name & $2.70(1.45)$ & $0-4$ & $0-4$ \\
\hline Appointment & $2.58(1.52)$ & $0-4$ & $0-4$ \\
\hline Belongings & $6.04(2.03)$ & $2-8$ & $0-8$ \\
\hline RBMT Standardized total & $33.26(5.17)$ & $21-44$ & $0-44$ \\
\hline
\end{tabular}

NOTE: RBMT = Rivermead Behavioural Memory Test. 
TABLE 2:

Correlation Coefficients Between Predictors and Dependent Variable $(N=100)$

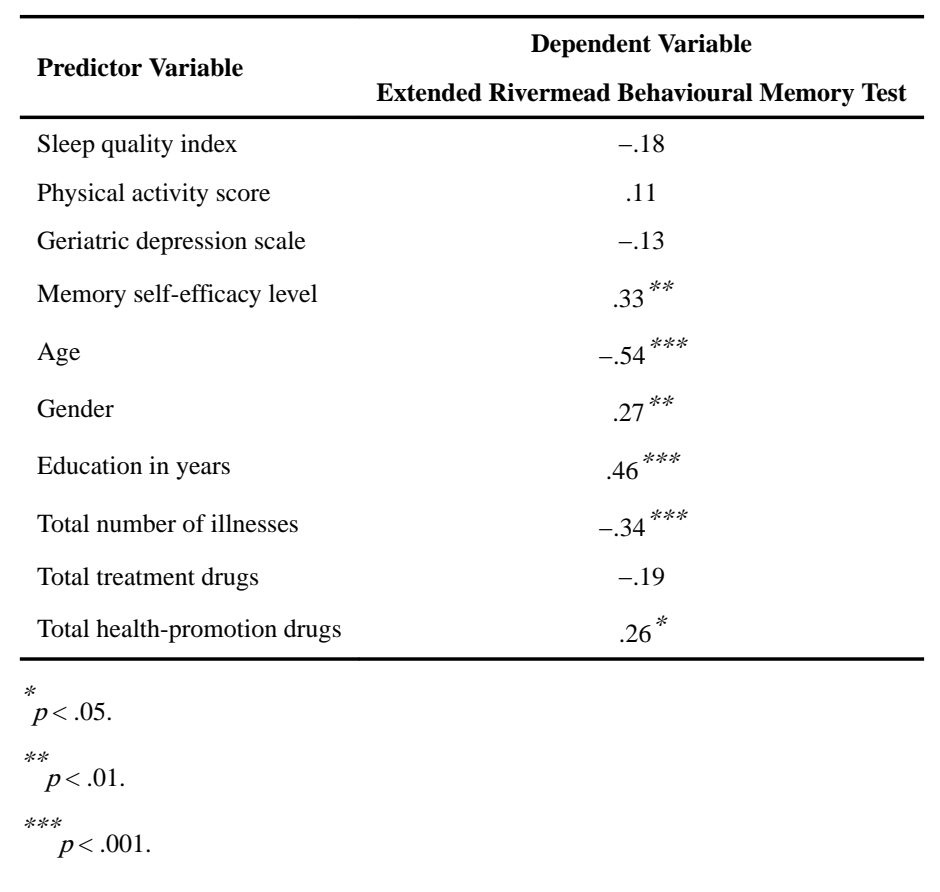




\section{TABLE 3:}

Simultaneous Multiple Regression Analysis of Four Independent Variables and Simultaneous Regression Analyses With Four Independent Variables and Six Control Variables on Memory Performance $(N=100)$

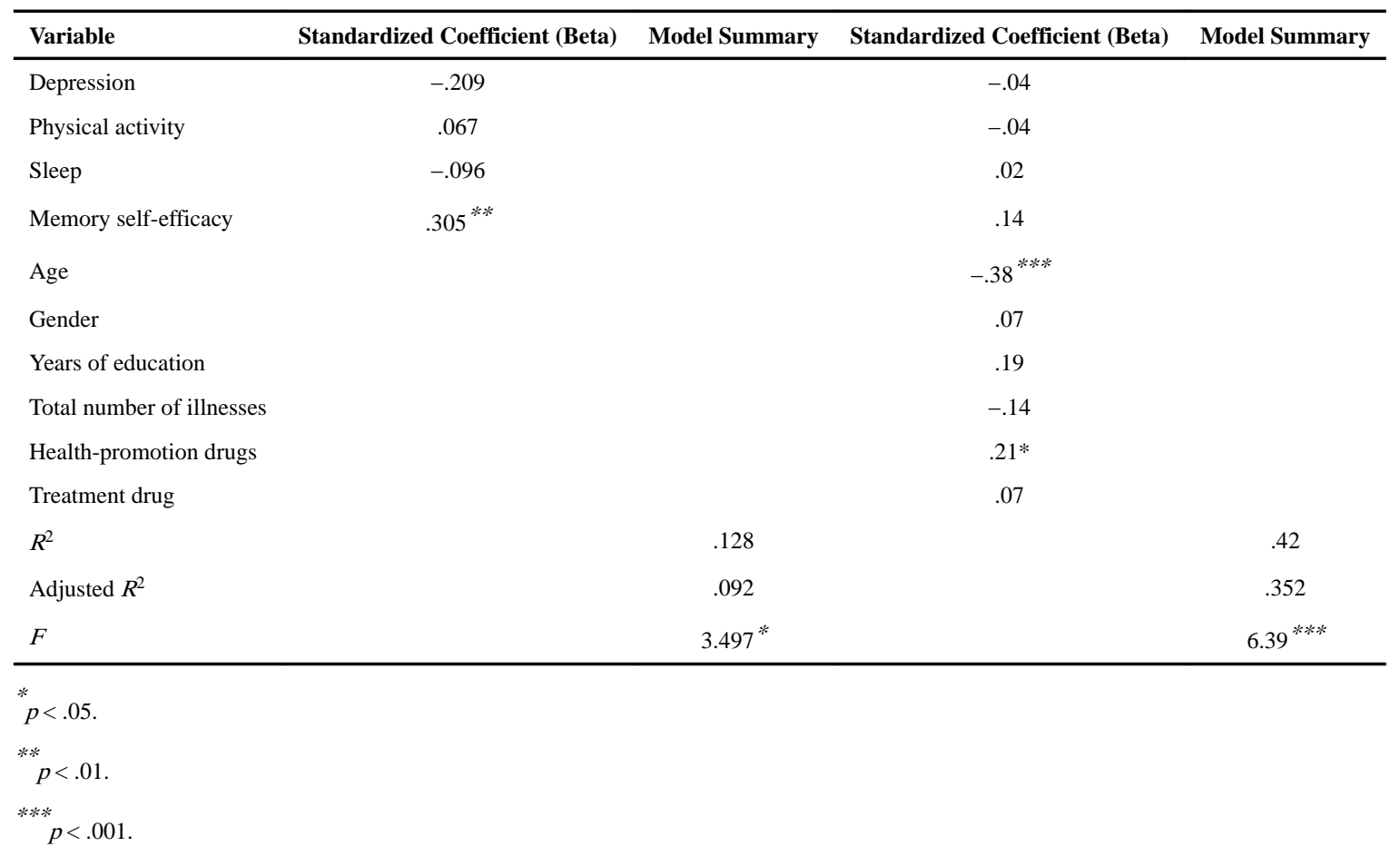

